\title{
Innovated PID pulse autotuner for fractional-order model set: the method of moments
}

\author{
Miloš Schlegel ${ }^{1}$ and Martin Čech $^{2}$
}

\begin{abstract}
Recently, a successful PID pulse autotuner based on model set approach was developed. Despite high reliability, several requirements for significant improvement have appeared after a decade of its industrial usage. Two of them are handled in this paper. Firstly, the set of a priori admissible processes was extended to fractional-order all-pole models to cover wider range of real plants. Secondly, a new parameter affecting the model set span was introduced. It helps markedly the practitioners to reach the proper robustness/performance ratio especially in the case when the initial controller tuning is too conservative. The authors believe that the new theory will be soon transferred to industrial practice.
\end{abstract}

\section{INTRODUCTION}

The producers of compact controllers and control systems aim at improving their products by intelligent features that shorten the time of installing into production. Clearly, such features should be serviceable without high-level knowledge of control theory. In particular, a huge effort is put into automatic PID controller tuning. The built-in function should reliable tune the controller for all typical industrial processes. It is a challenging problem which is not solved yet. The renowned studies estimate that $70 \%$ of PID loops are not well tuned, the controllers work with default parameters or even in manual mode. One of the reasons is that the PID producers prefer empirical methods instead of exact math theory [3], [10], [14].

In this paper, a novel exact approach is indicated which overcomes well known drawbacks of empirical methods (see e.g. [9] for nice overview) while the simplicity is preserved. The key paradigm is the model set which is defined as a set of all processes satisfying two types of information: $a$ priori assumption about the process candidate models and information get from identification experiment.

Remind, that in modern robust control theory, it is usually assumed that the frequency response of the "true" system lies for each frequency on regions of the complex plane [15]. These regions, called value sets, quantify the amount of model uncertainty at a given frequency. The problem of obtaining hard bounds of the value sets from experimental data has been considered before, see e.g. [13], [12], [8]. Although some of these methods are very sophisticated and general, none of them gives tight bounds for the case where only few characteristic numbers are mined from the

1 Miloš Schlegel is with the Faculty of Applied Sciences/NTIS, University of West Bohemia, Pilsen, Czech Republic schlegel@kky.zcu.cz

2 Martin Čech is with the Faculty of Applied Sciences/NTIS, University of West Bohemia, Pilsen, Czech Republic mcech@kky.zcu.cz

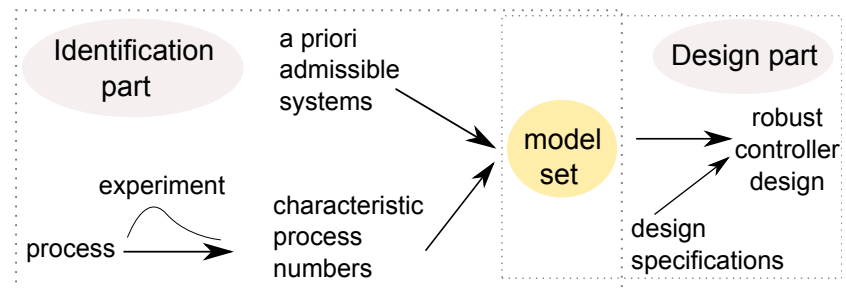

Fig. 1: General idea of the model set approach

identification experiment because of the minimum a priori information assumed.

The a priori information used in this paper separates from all possible linear systems relatively small set of allpole fractional processes; see [7]. However, such set is wide enough to cover majority of real plants (even with deadtime or stable zeros). Next, it is assumed that the simple rectangle pulse or step test is initiated by the controller. By processing the plant output, first three moments of process transfer function are obtained. All a priori admissible systems consistent with experimental data create a model set. The robust controller design resides on finding a controller satisfying frequency-domain design specifications for any process belonging to the model set and which minimizes certain optimization criterion, simultaneously. Such systematic PID tuning procedure for fractional all-pole models has not been done before, up to the authors' knowledge. Note, that the authors have used similar approach to develop a relay based PID tuner recently [18], [16].

The rest of the paper is organized as follows: Section II summarizes the problem formulation. In Section III, the parameterization of extremal processes is given. Section IV provides the results of numeric PID controller design. Section $\mathrm{V}$ describes the second generation of robust PID autotuner. Conclusions and ideas for future work are given in Section VI.

\section{PROBLEM FORMULATION}

Consider the feedback control loop with PID controller

$$
C(s)=K\left(1+\frac{1}{T_{i} s}+\frac{T_{d} s}{\frac{T_{d}}{N} s+1}\right),
$$

where $K, T_{i}$ and $T_{d}$ are proportional gain, integral and derivative time constant, respectively. A fixed parameter $N$ determines the time constant of a derivative term filter. Our aim is to design free parameters $K>0, T_{i}>0, T_{d}>0$ in such a way that the closed loop meets design requirements 
for all processes belonging to the model set. Further, let us state the problem more precisely:

\section{A. A priori admissible systems}

It was shown in [7] that to cover the huge number of real processes, one has a priori to consider the fractional-order transfer function in the form

$$
P(s)=\frac{K}{\prod_{i=1}^{p}\left(\tau_{i} s+1\right)^{n_{i}}},
$$

where $p$ is arbitrary integer number and $K, \tau_{i}, n_{i} i=1$, $2, \ldots, p$ are positive real numbers. The transfer function (2) describes very well the majority of essentially monotone processes (see [2] for definition).

Remark 1: If $p \rightarrow \infty$ then the set of all transfer functions (2) contains also processes with dead time and approximates well several processes with stable zeros ([20]) or with transcendent transfer functions (like heat transfer, chemical processes, etc., [3]).

\section{B. Characteristic numbers - experimental data}

Three-parameter time domain process description is well accepted in the control community. The authors' previous works vindicate the usage of first three moments $m_{i}$ of the impulse response $h(t)$ instead of numbers obtained from the step response using its tangent line in the inflexion point. The application of time moments in control appeared firstly in [11]. They are defined as

$$
m_{i}=\int_{0}^{\infty} t^{i} h(t) \mathrm{d} t, \quad i=0,1,2
$$

and may be converted to another more suitable group of numbers $\left\{\kappa, \mu, \sigma^{2}\right\}[17]$ defined as

$$
\begin{gathered}
\kappa=\int_{0}^{\infty} h(t) \mathrm{d} t=m_{0}, \quad \mu=\frac{\int_{0}^{\infty} t h(t) \mathrm{d} t}{\int_{0}^{\infty} h(t) \mathrm{d} t}=\frac{m_{1}}{m_{0}}, \\
\sigma^{2}=\frac{\int_{0}^{\infty}(t-\mu)^{2} h(t) \mathrm{d} t}{\int_{0}^{\infty} h(t) \mathrm{d} t}=\frac{m_{2}}{m_{0}}-\frac{m_{1}^{2}}{m_{0}^{2}} .
\end{gathered}
$$

It can be proved [5] that for transfer function (2) it holds

$$
\kappa=K, \quad \mu=\sum_{i=1}^{p} \tau_{i} n_{i}, \quad \sigma^{2}=\sum_{i=1}^{p} \tau_{i}^{2} n_{i} .
$$

From a control point of view, $\kappa$ is equal to process static gain, $\mu$ and $\sigma$ represent the residual time constant and reaction time, respectively. Without loss of generality, the process can be normalized in gain and time, thus $\bar{\kappa}=1$ and $\bar{\mu}=1$. The remaining parameter $\bar{\sigma}^{2}=\sigma^{2} / \mu^{2}$ then has a meaning similar to normalized dead time. It shapes the step response from first order to pure dead time process as shown in Fig. 2. The idea of normalization and process description by normalized dead-time is used quite frequently, e.g. in [14].

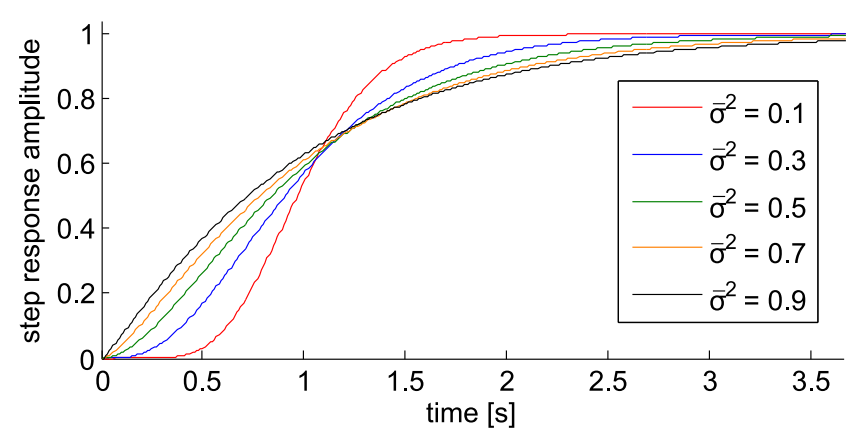

Fig. 2: Step response shaping by parameter $\bar{\sigma}^{2}$

Remark 2: The impulse response moments (3) or equivalently the numbers (4) can be obtained from the process step response as shown in Section IV. They may be also estimated from process input/ouput data. As they integral character (3), their values are not very sensitive to common noises in measured signal.

Assumption 1: In the following let us assume that we have measured precisely the numbers (4) and no other information about the process is available.

\section{Model set, extremal, vertex and ultimate processes}

To make the paper more self-contained let us briefly remind basic definitions and lemmas.

Definition 1 (Model set): The transfer function $P(s)$ is admissible if and only if

(i) $P(s)$ is in the form (2), $n_{i} \geq m, \forall i, \sum_{i=1}^{p} n_{i} \leq n$, where $n \in \mathbb{R}^{+}$is the total order of the process and $m \in \mathbb{R}^{+}$is the minimum allowed order of each fractional pole.

(ii) $P(s)$ is consistent with experimental data, thus fulfills (5). The set of all admissible transfer functions will be called model set and denoted as $\mathcal{S}^{n, m}\left(\kappa, \mu, \sigma^{2}\right)$.

Lemma 1: Let $n \geq 2 m$, then the model set $\mathcal{S}^{n, m}\left(\kappa, \mu, \sigma^{2}\right)$ is not empty if and only if

$$
\frac{1}{n} \leq \frac{\sigma^{2}}{\mu^{2}} \leq \frac{1}{m}
$$

The proof is omitted for brevity. If the inequality (6) is satisfied then the model set contains for given characteristic numbers $\kappa, \mu, \sigma^{2}$ infinite number of processes. Fortunately, these processes create after mapping into frequency domain a connected area called value set for each frequency $\omega>0$.

Definition 2 (Value set): The set $\mathcal{V}_{\omega}^{n, m}\left(\kappa, \mu, \sigma^{2}\right)=$ $=\left\{P(\mathrm{j} \omega): P(s) \in \mathcal{S}^{n, m}\left(\kappa, \mu, \sigma^{2}\right)\right\}$ will be called the value set of $\mathcal{S}^{n, m}\left(\kappa, \mu, \sigma^{2}\right)$ at the frequency $\omega>0$.

The value set boundary is generated by so called extremal transfer functions.

Definition 3 (Extremal transfer function): The admissible transfer function $P(s) \in \mathcal{S}^{n, m}\left(\kappa, \mu, \sigma^{2}\right)$ will be called extremal, if there exists $\omega>0$ such, that $P(\mathrm{j} \omega) \in$ $\partial \mathcal{V}_{\omega}^{n, m}\left(\kappa, \mu, \sigma^{2}\right)$, where $\partial \mathcal{V}_{\omega}^{n, m}\left(\kappa, \mu, \sigma^{2}\right)$ denotes the value set boundary in complex plane. Let us denote the set of all extremal transfer functions as $\mathcal{S}_{E}^{n, m}\left(\kappa, \mu, \sigma^{2}\right)$.

Remark 3: For the a priori assumption (2) and condition (5) the set $\mathcal{S}_{E}^{n, m}\left(\kappa, \mu, \sigma^{2}\right)$ is independent on frequency $\omega$. 


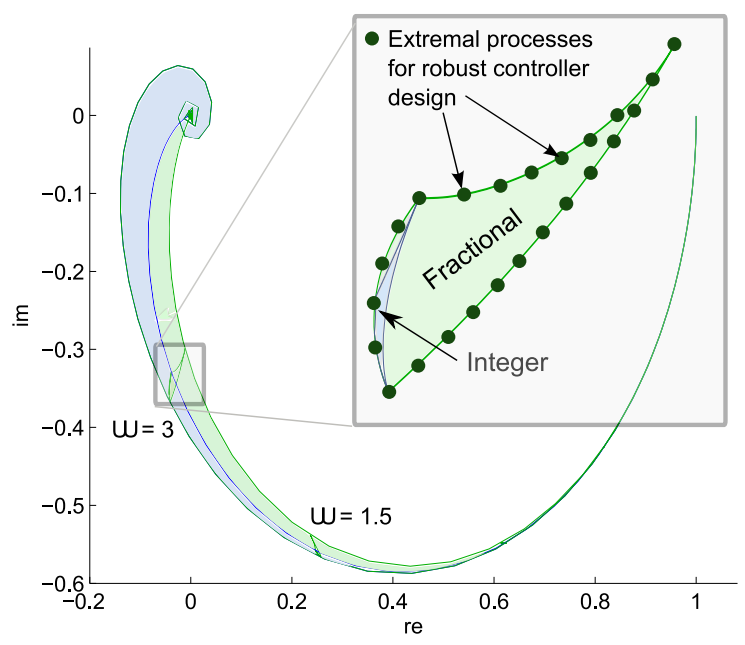

Fig. 3: Comparison of integer (blue) and fractional order (green) model set uncertainty in frequency domain $\left(\sigma^{2}=\right.$ $0.7)$; detailed boundary of $\mathcal{V}_{3}^{\infty, 1}(1,1,0.7)$.

The value set boundary is composed of finite number of smooth curves. In authors previous work [17], the analytical relations for computing value set boundaries (extremal processes) were derived for integer-order (IO) systems. For the new PID autotuner, the same was done for fractionalorder processes. In Fig. 3, one can examine that omitting fractional-order processes reduces the uncertainty markedly ${ }^{1}$.

Remark 4: It is acceptable to define the minimum pole order as $m=1$. Processes of order less than one do not have an equivalent in the real word. Besides, they extend more and more the model set uncertainty. On the contrary, the total process order $n$ defines the model set uncertainty and can be used as a parameter assessing the robustness/performance ratio. The first autotuner generation was derived just for the case $n \rightarrow \infty$ which often led to very sluggish control. It is also worth to mention, that this approach is much less conservative compared to popular $H_{\infty}$ techniques where the uncertainty has a circle shape for each frequency $\omega$.

Definition 4 (Robust controller design task): Assume that it is given fixed real $n>2$ and $\sigma^{2}$ satisfying (6). Find the parameters of controller (1) such that

$$
I \triangleq \frac{T_{i}}{K} \rightarrow \min
$$

while the following two conditions are satisfied for any $P(s) \in \mathcal{S}^{n, 1}\left(\bar{\sigma}^{2}\right)$ :

(i) (Stability) Nyquist plot $L(\mathrm{j} \omega) \triangleq C(\mathrm{j} \omega) P(\mathrm{j} \omega)$ fulfills closed loop stability condition, i.e. it does not encircle critical point $(-1, \mathrm{j} 0)$.

(ii) (Stability margin) Nyquist plot $L(\mathrm{j} \omega)$ lies outside a circle with a center $c \in \mathbb{C}$ and radius $r \in \mathbb{R}^{+}$, i.e.

$$
\forall \omega \geq 0: L(\mathrm{j} \omega) \notin U(c, r),
$$

\footnotetext{
${ }^{1}$ The dynamics of real distributed parameter processes is closed to fractional. Hence considering only $I O$ processes in robust design may lead to non-satisfactory closed loop behavior.
}

where $U(c, r) \triangleq\{s \in \mathbb{C}:|s-c|<r\}$ is an open circle in complex plane.

Remark 5: It is well known that by condition (ii) one can express various requirements. If the sensitivity function needs to be restricted as

$$
\sup _{\omega}\left|\frac{1}{1+L(j \omega)}\right| \leq M_{s}
$$

then one choose

$$
c=-1, \quad r=\frac{1}{M_{s}} .
$$

Similarly, for complementary sensitivity function restriction

$$
\sup _{\omega}\left|\frac{L(j \omega)}{1+L(j \omega)}\right| \leq M_{t}
$$

then one choose

$$
c=\frac{M_{t}^{2}}{1-M_{t}^{2}}, \quad r=\frac{M_{t}}{\left|M_{t}^{2}-1\right|} .
$$

Detailed discussion can be found in [4].

Lemma 2: Let $L(\mathrm{j} 0) \notin U(c, r)$ for any $P(s) \in$ $\mathcal{S}^{n, m}\left(\kappa, \mu, \sigma^{2}\right)$. Then the property $\chi$ is is fulfilled for any $P(s) \in \mathcal{S}^{n, m}\left(\kappa, \mu, \sigma^{2}\right)$ if and only if $\chi$ is fulfilled for all $P(s) \in \mathcal{S}_{E}^{n, m}\left(\kappa, \mu, \sigma^{2}\right)$

The proof is simple and is omitted due to the limited paper space.

\section{EXTREMAL PROCESSES PARAMETERIZATION}

Theorem $1\left(\partial \mathcal{V}_{\omega}\right.$ parameterization): Let $n \geq 3 m, m>0$, then for arbitrary $\omega>0$ any point of the boundary $\partial \mathcal{V}_{\omega}$ of the value set created by the model set $\mathcal{S}^{n, m}\left(1,1, \sigma^{2}\right)$ can be generated by process which can be expressed either in the form (holds true for $\frac{1}{n} \leq \sigma^{2} \leq \frac{1}{m}$ )

$$
P(s)=\frac{1}{\left(\tau_{1}(\alpha) s+1\right)^{n_{1}(\alpha)}\left(\tau_{2}(\alpha) s+1\right)^{n_{2}(\alpha)}},
$$

where

$$
\begin{aligned}
\tau_{1}(\alpha) & =\frac{1-\sqrt{\frac{n_{2}}{n_{1}}\left(\sigma^{2}\left(n_{1}+n_{2}\right)-1\right)}}{\left(n_{1}+n_{2}\right)}, \\
\tau_{2}(\alpha) & =\frac{1+\sqrt{\frac{n_{1}}{n_{2}}\left(\sigma^{2}\left(n_{1}+n_{2}\right)-1\right)}}{\left(n_{1}+n_{2}\right)}
\end{aligned}
$$

and $n_{1}, n_{2}$ are determined by three different ways:

(i) $n_{1}(\alpha)=m, n_{2}(\alpha)=\alpha$,

$\alpha \in\left[\max \left\{m, \frac{1-m \sigma^{2}}{\sigma^{2}}\right\}, \min \left\{n-m, \frac{1}{\sigma^{2}}\right\}\right]$

(ii) $n_{1}(\alpha)=\alpha, n_{2}(\alpha)=m$,

$$
\alpha \in\left[\max \left\{m, \frac{1-m \sigma^{2}}{\sigma^{2}}\right\}, n-m\right]
$$

(iii) $n_{1}(\alpha)=n-\alpha, n_{2}(\alpha)=\alpha$, $\alpha \in\left[m, \min \left\{n-m, \frac{1}{\sigma^{2}}\right\}\right]$,

or in the form (holds true only for $\frac{1}{n} \leq \sigma^{2} \leq \frac{1}{n-2 m}$ )

$$
P(s)=\frac{1}{\left(\tau_{1}(\alpha) s+1\right)^{m}\left(\tau_{2}(\alpha) s+1\right)^{m}\left(\tau_{3}(\alpha) s+1\right)^{n-2 m}},
$$




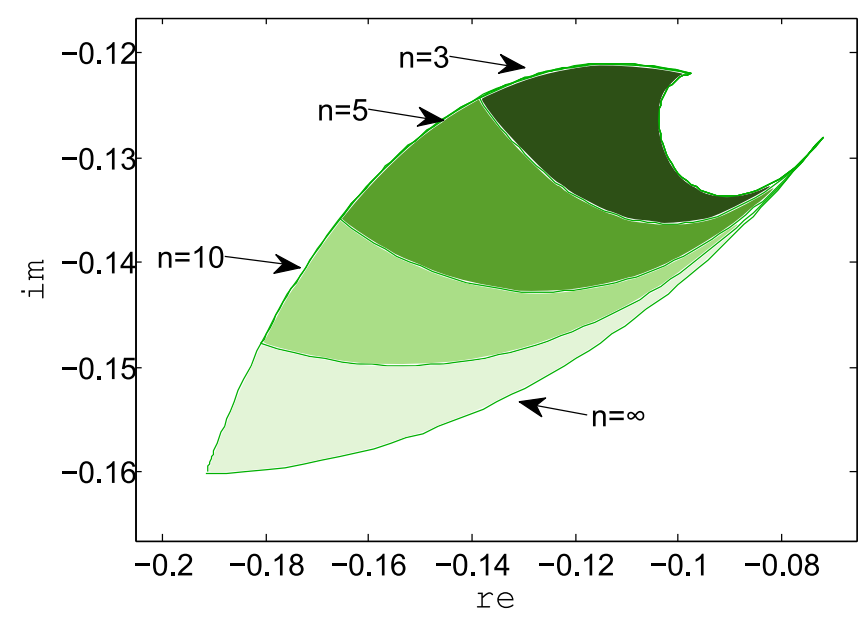

Fig. 4: Illustration of increasing span of the value set $\mathcal{V}_{5}^{n, 1}(1,1,0.6)$ related to maximum process order $n$.

where

$\begin{aligned} \tau_{1}(\alpha) & =\frac{1-\alpha(n-2 m)-\sqrt{(n-2 m) \alpha(2-n \alpha)+2 m \sigma^{2}-1}}{2 m} \\ \tau_{2}(\alpha) & =\frac{1-\alpha(n-2 m)+\sqrt{(n-2 m) \alpha(2-n \alpha)+2 m \sigma^{2}-1}}{2 m} \\ \tau_{3}(\alpha) & =\alpha,\end{aligned}$

for the parameter $\alpha$ it holds

$$
\begin{aligned}
& \alpha \in\left[a_{1}, b\right] \quad \text { pro } \frac{1}{n} \leq \sigma^{2} \leq \frac{1}{n-m} \\
& \alpha \in\left[a_{2}, b\right] \quad \text { pro } \quad \frac{1}{n-m} \leq \sigma^{2} \leq \frac{1}{n-2 m} \\
& a_{1}=\frac{(n-m)+\sqrt{n(n-m)\left(n \sigma^{2}-1\right)}}{n(n-m)} \\
& a_{2}=\frac{\left.(n-2 m)+\sqrt{m(n-2 m)\left[(n-m) \sigma^{2}-1\right.}\right]}{(n-m)(n-2 m)} \\
& b=\frac{(n-2 m)+\sqrt{2 m(n-2 m)\left(n \sigma^{2}-1\right)}}{n(n-2 m)}
\end{aligned}
$$

The proof is omitted for brevity. Theorem 1 is the main theoretical result of the paper. It allows to parameterize effectively all extremal processes which can play an active role in robust controller design.

An example of value set boundary is shown in Fig. 3. For each frequency $\omega$, it is a closed curve consisting from three arcs that correspond to particular transfer function forms stated in Theorem 1. Evidently, the extremal processes creating the boundary are the most important when solving optimal controller design procedure as the contact point between $C(\mathrm{j} \omega) \mathcal{V}_{\omega}^{n, m}\left(\kappa, \mu, \sigma^{2}\right)$ and $U(c, r)$ is at some frequency $\omega>0$ generated by some $P(s) \in \mathcal{S}_{E}^{n, m}\left(\kappa, \mu, \sigma^{2}\right)$.

\section{NuMERIC SOLUTION OF ROBUST PID CONTROLLER DESIGN}

In this Section, let us describe the results of optimization procedure which is based on generalized robustness regions method [21], [19], [1] and the claims of Theorem 1 and

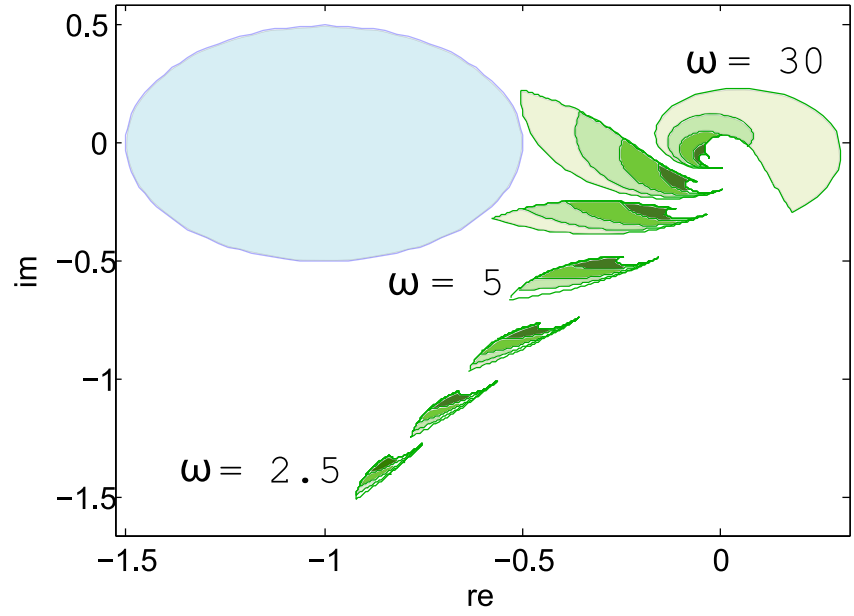

Fig. 5: Nyquist plot uncertainty depicted for $(n \in\{3,5,10, \infty\})$ for various frequencies $\omega \in$ $\{2.5,3,4,5,8,12,30\}$. The controller is the optimal one respecting $M_{s}=2$.

Lemma 2. The optimization problem can be solved effectively for arbitrary $n \geq 2$ and even for $n \rightarrow \infty$. Without loss of generality, the problem could be restricted to the model set normalized in gain and time denoted as $\mathcal{S}^{n, 1}\left(\bar{\sigma}^{2}\right)$ where $\bar{\sigma}^{2} \in(0,1)$ and $n \in(2, \infty)$. Consequently, let us denote the computed controller parameters as $\bar{K}, \bar{T}_{i}, \bar{T}_{d}$. Further assume that for PID parameters it holds $N=10$ and $T_{i} / T_{d}=4$ and the sensitivity function is restricted by the circle described by (10) where $M_{s}=2.0$. The optimal parameters $\bar{K}, \bar{T}_{i}$ can be for each $n$ approximated by the function in the form

$$
f_{n}(\bar{\sigma})=a_{0} \mathrm{e}^{a_{1} \bar{\sigma}+a_{2} \bar{\sigma}^{2}+a_{3} \bar{\sigma}^{3}+a_{4} \bar{\sigma}^{4}}
$$

where the coefficient $a_{0}, a_{1}, \ldots, a_{4}$ are stored in internal controller tables (example shown in Table I).

TABLE I: Example of parameter values for $n=\infty$

\begin{tabular}{c|cc} 
& $\bar{K}$ & $\bar{T}_{i}$ \\
\hline$a_{0}$ & 0.46 & 0.33 \\
$a_{1}$ & -2.79 & 25.8 \\
$a_{2}$ & 19.2 & -6.74 \\
$a_{3}$ & -27.7 & 11.2 \\
$a_{4}$ & 14.7 & -7.87
\end{tabular}

Finally, a new tuning parameter $\delta$ can be introduced which maps model set order $n$ to the 'user' interval $(0,1)$ as follows

$$
\delta=\frac{2}{n} \text {. }
$$

Such parameter helps to handle easily the robustness/performance tradeoff. The values of controller parameters $K$ and $T_{i}$ depending on parameter $\delta$ can be checked in Fig. 6 and 7.

\section{SECOND Generation OF ROBUSt PID AUTOTUNER}

The complete automatic tuning procedure will be described in this Section. Firstly, let us explain how the characteristic numbers $\kappa, \mu, \sigma^{2}$ can be obtained on real plant. It 


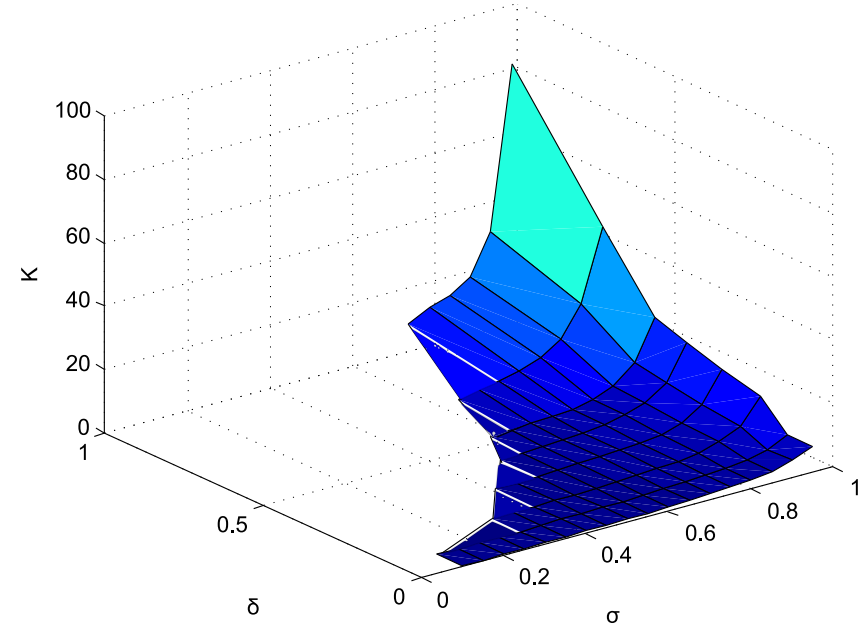

Fig. 6: Controller gain $K$ depending on $\sigma$ and $\delta$

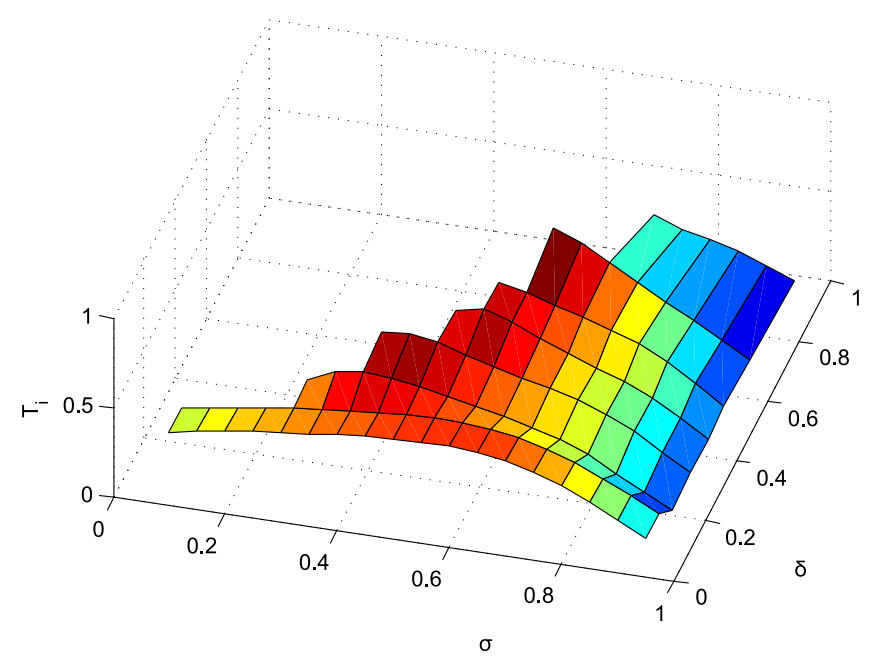

Fig. 7: Controller integral time constant $T_{i}$ depending on $\sigma$ and $\delta$

flows out from (4) that they can be determined by integrating process impulse response $h(t)$. Clearly, different approach has to be adopted in practice. The following lemma will help to solve that issue:

Lemma 3: Let the process $P_{i}(s)$ have a residual time constant $\mu_{i}$ and a reaction time $\sigma_{i}, i=1,2, \ldots, m$ then the serial connection

$$
P(s)=P_{1}(s) P_{2}(s) \cdots P_{m}(s)
$$

has a residual time constant

$$
\mu=\mu_{1}+\mu_{2}+\ldots \mu_{m}
$$

and for the reaction time, it holds

$$
\sigma^{2}=\sigma_{1}^{2}+\sigma_{2}^{2}+\ldots \sigma_{m}^{2}
$$

The proof is omitted for brevity. Further, consider a serial connection $H(s)=T(s) P(s)$ where

$$
T(s)=\frac{1}{s}\left(1-e^{-D s}\right) .
$$

When a Dirac pulse is applied as an input of $T(s)$ then a rectangle pulse with unit amplitude and width equal to $D$ excites $P(s)$. Using (4), one can determine the characteristic numbers $\kappa_{H}, \mu_{H}$ and $\sigma_{H}$ of a hypothetical serial connection $H(s)$ from process reaction to respective rectangular pulse. Consequently, using Lemma 3 , the process characteristic numbers can be determined as

$$
\begin{aligned}
\kappa & =\frac{\kappa_{H}}{D}, \\
\mu & =\mu_{H}-\frac{D}{2}, \\
\sigma^{2} & =\sigma_{H}^{2}-\frac{D^{2}}{12} .
\end{aligned}
$$

Using (24) and internal Tables, the optimal controller parameters can be determined for various uncertainty span $n$ as follows:

1) After the identification experiment, the normalized $\bar{\sigma}$ is computed from the relation $\bar{\sigma}^{2}=\sigma^{2} / \mu^{2}$.

2) The normalized controller parameters $\bar{K}, \bar{T}_{i}$ are gained from the internal tables

3) The controller parameters are denormalized as follows $K=\kappa \bar{K}, T_{i}=\mu \bar{T}_{i}, T_{d}=\mu \bar{T}_{d}$.

In Fig. 8 one can see the autotuner performance on a fractional process with transfer function

$$
P(s)=\frac{1}{(2.58 s+1)^{5.6}(5.54 s+1)} .
$$

Note, that for the time domain simulation, the fractionalorder system must always be approximated, e.g. using optimal integer-order approximations described in [6].

The first autotuner generation is successfully employed in numerous stand-alone compact PID controllers as well as higher level commercial control systems. Hence the authors believe that the new PID tuner generation described in this paper will appear in industrial practise soon.

\section{CONCLUSION}

The next generation of recently developed PID pulse autotuner was reported in this paper. At present, the autotuner takes also fractional order all-pole models into account. Moreover, the new tuning parameter was introduces. It helps to handle the robustness/performance trade-off especially when the default tuning seems to be too conservative. Point out that these research results can be used by engineers without deep knowledge of control theory. The pulse experiment is much shorter compared to traditional relay experiment. On the contrary, it requires more propitious signal/noise ratio.

\section{ACKNOWLEDGEMENT}

This work was supported by the Technology Agency of the Czech Republic - project No. TA02010152 and by the European Regional Development Fund (ERDF), project NTIS New Technologies for Information Society, European Centre of Excellence, CZ.1.05/1.1.00/02.0090. The support is gratefully acknowledged. 


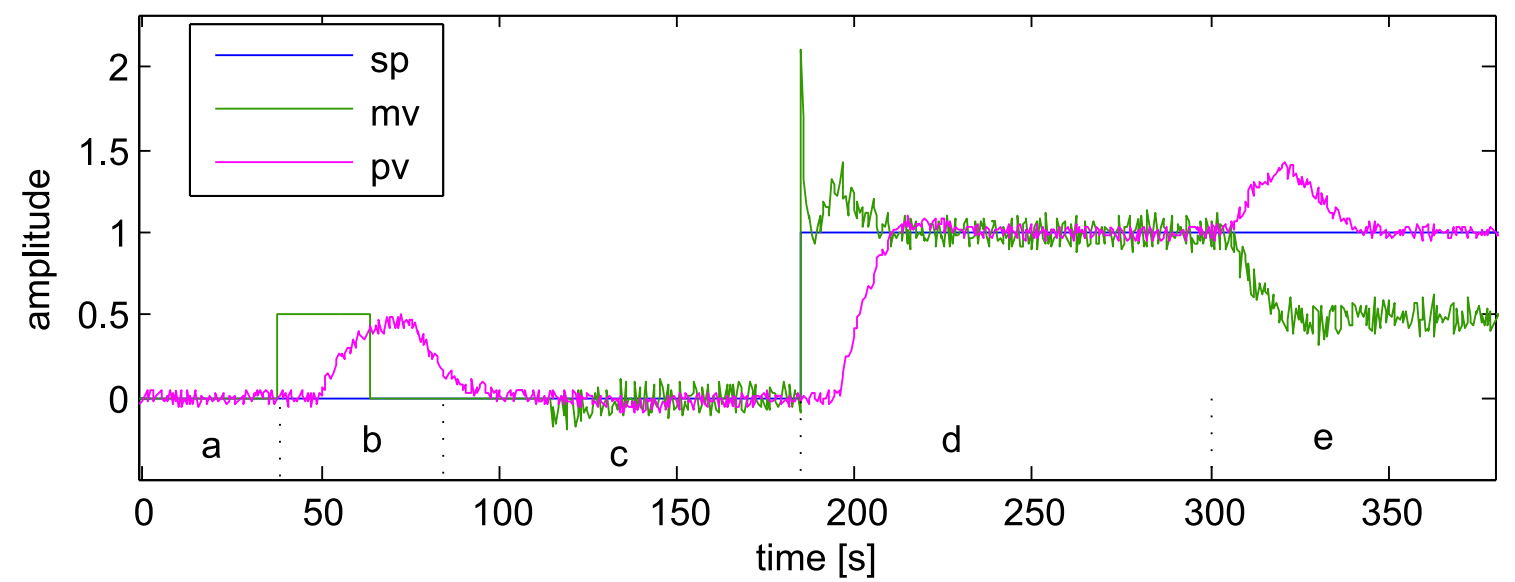

Fig. 8: Application of PID autotuner to the static monotone process with significant time delay. Signals: pv - process variable, sp - setpoint, mv - manipulated value. Autotuning phases: a - drift and noise estimation, b - identification experiment, c autotuner in automatic mode, $\mathrm{d}$ - setpoint step response, e - load disturbance step response

\section{REFERENCES}

[1] K.J. Åström and T. Hägglund. The future of PID control. Control Engineering Practice, 9:1163-1175, 2001.

[2] K.J. Åström and T. Hägglund. Revisiting the Ziegler - Nichols step response method for PID control. Journal of Process Control, 14(6):635 - 650, 2004.

[3] K.J. Åström and T. Hägglund. Advanced PID control. ISA Instrumentation, Systems and Automation Society, 2006. ISBN: 155617-942-1.

[4] K.J. Åström, H. Panagopoulos, and T. Hägglund. Design of PI controllers based on non-convex optimization. Automatica, 34(5):585 $-601,1998$.

[5] M. Čech. Robust controller design for fractional systems. Ph.D. thesis, Univ. of West Bohemia, Pilsen, 2008.

[6] M. Čech and M. Schlegel. Optimal continuous approximation of basic fractional elements: Theory and applications. In Proceedings of the IEEE Conference on Decision and Control, pages 7051-7056, December 2011.

[7] A.C. Charef, H.H. Sun, Y.Y. Tsao, and B. Onaral. Fractal system as represented by singularity function. IEEE Trans. on Automatic Control, 37:1465-1470, 1992.

[8] A.J. Helmicki, C.A. Jacobson, and C.N. Nett. Control oriented system identification: a worst case deterministic approach in $H_{\infty}$. IEEE Transactions on Automatic Control, 36:1163-1176, 1991.

[9] M.T. Ho, A. Datta, and S.P. Bhattacharyya. A linear programming characterization of all stabilizing PID controllers. In Proceedings of the Amarican Control Conference, pages 3922-3928, Albuquerque, 1997.

[10] A. Leva. Model-based tuning: the very basic and some useful techniques. Journal A, 42(3):14-22, 2001.

[11] N. Maamri and J.-C. Trigeassou. PID design for time delayed systems by the method of moments. In European Control Conference, Groningen Holland, 1993.

[12] M. Milanese, J. Norton, H. Piet-Lahanier, E. Walter, and Eds. Bounding approaches to system identification. Plenum, New York, 1996.

[13] H. Nijmeijer and A. Schaft. Nonlinear dynamical control systems. Springer-Verlag, New York, 1990.

[14] F. Padula and A Visioli. Tuning rules for optimal PID and fractionalorder PID controllers. Journal of Process Control, 21(1):69 - 81, 2011.

[15] H. Rotstein, N. Galperin, and P. Gutman. Set membership approach for reducing value sets in the frequency domain. IEEE Transactions on Automatic Control, 43:1346-1350, 1998.

[16] M. Schlegel. Exact revision of the Ziegler-Nichols frequency response method. In Proc. of IASTED Int. Conf. on control and applications, Cancun, Mexico, 2002.

[17] M. Schlegel, P. Balda, and M. Štětina. Robust PID autotuner - method of moments. Automatizace, 46(4), 2003.
[18] M. Schlegel and M. Čech. Computing value sets from one point of frequency response with applications. In Proceedings of IFAC World Congress 2005, Praque, Czech Republic, July 2005.

[19] Z. Shafiei and A.T. Shenton. Frequency-domain design of PID controllers for stable and unstable systems with time delay. Automatica, 33:2223-2232, 1997.

[20] S. Skogestad. Simple analytic rules for model reduction and PID controller tuning. Journal of Process Control, 13:291-309, 2003

[21] M. Čech and M. Schlegel. Generalized robust stability regions for fractional PID controllers. In Proceedings of the IEEE International Conference on Industrial Technology, pages 76-81, 2013. 